\title{
Endobronchial aspergilloma: A case report and literature review
}

\author{
DONGDONG HUANG ${ }^{1 *},{\text { BING } \text { LI }^{2 *}, \text { HAIQING CHU }}^{2}$, ZHEMIN ZHANG $^{2}$, QIUHONG SUN $^{2}$, \\ LAN ZHAO $^{2}$, LIYUN XU ${ }^{2}$, LI SHEN ${ }^{2}$, TAO GUI ${ }^{2}$, HUIKANG XIE $^{3}$ and JUN ZHANG ${ }^{4}$ \\ ${ }^{1}$ Department of Clinical Medicine, Tongji University School of Medicine, Shanghai 200092; Departments of \\ ${ }^{2}$ Respiratory Medicine and ${ }^{3}$ Pathology, Shanghai Pulmonary Hospital, Tongji University School of Medicine, \\ Shanghai 200433, P.R. China; ${ }^{4}$ Division of Hematology, Oncology and Blood \& Marrow \\ Transplantation, Department of Internal Medicine, Holden Comprehensive Cancer Center, \\ University of Iowa Carver College of Medicine, Iowa, IA 52242, USA
}

Received March 6, 2016; Accepted March 23, 2017

DOI: $10.3892 /$ etm.2017.4540

\begin{abstract}
The present study aimed to investigate the clinical and radiological characteristics in addition to the bronchoscopic appearance in patients with endobronchial aspergilloma (EBA). Clinical and radiological characteristics were analyzed alongside the bronchoscopic appearance in 17 patients with EBA diagnosed by bronchoscopy with histological examination. The present study assessed the relevant literature and 13 males and 4 females were included in the comparison, with a median age of 59. Associated diseases included 8 previous diagnoses of pulmonary tuberculosis $(47.6 \%), 4$ previous diagnoses lung cancer (23.5\%), 1 pulmonary resection $(5.9 \%)$ and 1 bronchial foreign body (5.9\%). The primary symptom was hemoptysis $(9 / 17,53 \%)$. Chest computed tomography (CT) indicated a markedly higher incidence of aspergillosis lesion in
\end{abstract}

Correspondence to: Professor Haiqing Chu or Professor Zhemin Zhang, Department of Respiratory Medicine, Shanghai Pulmonary Hospital, Tongji University School of Medicine, 507 Zhengmin Road, Shanghai 200433, P.R. China

E-mail: chu_haiqing@126.com

E-mail: zhemindoc@163.com

*Contributed equally

Abbreviations: EBA, endobronchial aspergilloma; CT, computer tomography; IPA, invasive pulmonary aspergillosis; CAN, chronic necrotizing aspergillosis; ABPA, allergic bronchopulmonary aspergillosis; SIA, semi-invasive aspergillosis; FDG, ${ }^{18} \mathrm{~F}$ deoxyglucose; FOB, fiberoptic bronchoscopy; DCMP, dilated cardiomyopathy; HT, hypertension; NSCLC, non-small cell lung cancer; ET, essential thrombocytosis; SDH, subarachnoid hemorrhage; ICH, intracranial hemorrhage; TB, tuberculosis; BW, bronchial washing; MTB, Mycobacterium tuberculosis; IV, intravenous; GGO, ground glass opacity; LLL, left lower lobe; LUL, left upper lobe; RML, right middle lobe; RUL, right upper lobe; $\mathrm{RBI}$, right bronchus intermedius

Key words: fungi, endobronchial aspergilloma, bronchoscopy the left lung $(13 / 17 ; 76.5 \%)$ compared with the right lung (4/17; $23.5 \%)$. CT manifestation included space occupying disease in 10 patients $(58.8 \%)$, aspergilloma in 3 patients (17.6\%), pneumonic consolidation in 2 patients $(11.8 \%)$ and ground glass opacity in 1 patient $(5.9 \%)$. Bronchoscopy examination identified masses in all 17 patients' bronchial lumen and 15 patients had endobronchial obstruction by necrotic material. The case presented in the current study demonstrated the merits of combining bronchosopic intervention with voriconazole. The dominant symptom of EBA was hemoptysis. Chest CT demonstrated that aspergillosis lesions were more frequently identified in the left lung compared with the right. EBA often occurs in individuals with underlying lung diseases, which cause lumen structural change or bronchial obstruction. EBA may be clearly diagnosed by bronchoscopy biopsy, although the potential for a co-exististing tumor requires consideration. Bronchoscopic intervention and anti-fungal therapy may have an advantage in the effective treatment of patients with EBA.

\section{Introduction}

Aspergillus spp. are a ubiquitous fungus in the environment, which are easily identified in soil, water and various types of decomposing organic matter (1). Inhalation of Aspergillus spores causes pulmonary aspergillosis, which is the most common form of aspergillus filamentous fungal infection, and is often diagnosed in patients with immune deficiencies or systemic diseases (2). Aspergillosis is currently divided into the following categories (3): i) Invasive pulmonary aspergillosis (IPA), a severe disease and major cause of mortality in severely immunocompromised patients; ii) chronic necrotizing aspergillosis (CNA), presents as a locally invasive disease and is observed primarily in patients who are mildly immunocompromised or have chronic lung disease; and iii) allergic bronchopulmonary aspergillosis (ABPA), a non-invasive hypersensitivity pulmonary disease caused by Aspergillus. Aspergilloma is a fungus ball that develops in a pre-existing cavity in lung parenchyma, which may be easily detected by X-ray and computed tomography (CT) scans (3). In addition, a novel category of aspergillosis with semi-invasive form (SIA) has been described (4). 

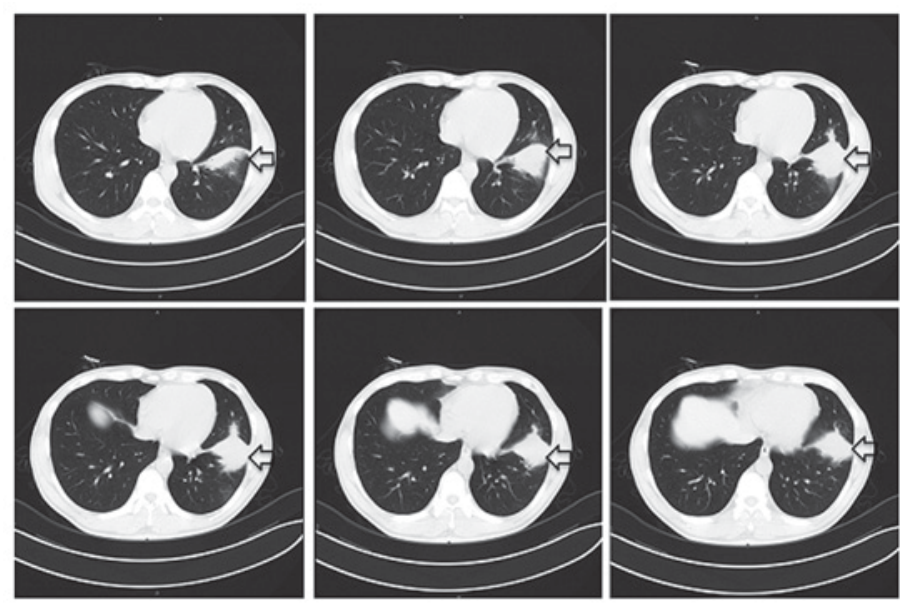

Figure 1. Chest computed tomography scan of a 48-year-old male patient revealed a spiculated mass and exudation in left lower lobe (arrow). The patient initially presented with a recurrent fever, cough, expectoration and bloody sputum lasting 3 months.

Endobronchial aspergilloma (EBA) is a rare disease, described as a massive non-invasive overgrowth of the aspergillus species in bronchial lumen, causing space-occupying lesions $(5,6)$. It is often incidentally diagnosed during bronchoscopy to evaluate the cause of hemoptysis $(5,6)$, and may be considered as another form of pulmonary aspergillosis. At present, there are very few reports regarding the characteristics of EBA. The present study aimed to investigate the clinical and radiological characteristics in addition to the bronchoscopic appearance in patients with EBA through a representative case from Shanghai Pulmonary Hospital affiliated to Tongji University (Shanghai, China) and a review of the current literature.

\section{Case report}

Study design. The present study reports a case of EBA with no prior treatment that was diagnosed at Shanghai Pulmonary Hospital affiliated to Tongji University, informed consent was also obtained from the patient. A retrospective analysis of the clinical and radiological characteristics and bronchoscopic results was also completed by combining this case with 16 other previously reported EBA cases (5-10). All patients we subjected to a chest CT, bronchoscopy and histological examination. The tissue used for histological examination was obtained via bronchoscopy biopsy of the mass in the trachea, avoiding necrotic tissue or secondary infection areas, with a volume of at least $0.5 \times 0.5 \times 0.2 \mathrm{~cm}$ and had a fresh non-necrotic color. The tissue was fixed using $10 \%$ neutral formaldehyde for $24 \mathrm{~h}$ at room temperature, routinely selected, embedded, sliced in continuous sections with a thickness of $4 \mu \mathrm{m}$ and hematoxylin-eosin (HE) stained. The test results and basic clinical data were collected. Primary evidence to confirm EBA were as follows: i) Presence of bronchoscopy-visible intraluminal mass and necrotic tissue; ii) characteristic appearance of Aspergillus species under microscopic examination, such as septate fungal hyphae bearing acute branching angles; iii) positive result of Aspergillus culture; and iv) following the elimination of other possibilities, such as tumors, foreign bodies, parasites or mucus by evaluating the patient's personal history, performing bronchoscopy and pathology examinations.
Representative case. A 48-year-old male was admitted to Shanghai Pulmonary Hospital affiliated to Tongji University due to a recurrent fever, cough, expectoration and bloody sputum lasting 3 months. The patient had a 20 -year history of smoking, and a 4-year history of diabetes and was undergoing diet therapy. On admission, he presented with normal vital signs. Physical examination of the chest revealed no evident crackles or wheezing. Complete blood count results were normal. Routine blood chemistry and tumor markers were all within normal limits. Mycodextranase and latex agglutination tests from the blood were negative. Human immunodeficiency virus antibody test was negative. Chemical luminescence of syphilis test was negative.

An intial CT scan of the chest revealed a spiculated mass with exudation in the left lower lobe (Fig. 1). ${ }^{18} \mathrm{~F}$ - deoxyglucose (FDG) dual-head coincidence single-photon emission computerized tomography (DHTC) indicated that the maximum tumor to non-tumor ratio was 11.63 and therefore, the possibility of lung cancer was not eliminated (Fig. 2). Initial fiberoptic bronchoscopy (FOB) examination (11) revealed a white necrotic mass in the medial anterior basal segment of left lower lobe, which completely obstructed the subsegmental bronchus (Fig. 3). Removal of the mass was attempted but was not successful as it was impacted in the narrow lumen. Notably, histopathological examination performed using HE staining of the bronchoscopic biopsied specimen was indicated to be negative. FOB was performed a second time 2 days later and a purulent material partially obstructing the lumen was identified at the same site. The lumen was finally unblocked following repetitive clearing of the necrotic material and sputum aspiration. The clearing work performed with a BF-1T260 bendable bronchoscopy (Olympus, Ishikawa, Japan) and biopsy forceps (Aohua, Shanghai, China), to cut and remove the tissue, $10 \mathrm{~min}$ a time for a total of 3 times. Histopathological examination of the bronchoscopic biopsied specimen indicated the presence of necrotic tissue and Aspergillus. Periodic acid-Schiff staining was performed and samples were exposed to $0.5 \%$ periodic acid for $5 \mathrm{~min}$, Schiffs' reagent for $15 \mathrm{~min}$ and then washed twice using $0.5 \%$ sodium metabisulfite for $1 \mathrm{~min}$ each time before bright green staining was performed for $10 \mathrm{sec}$ at room temperature. Furthermore, hexamine silver staining 


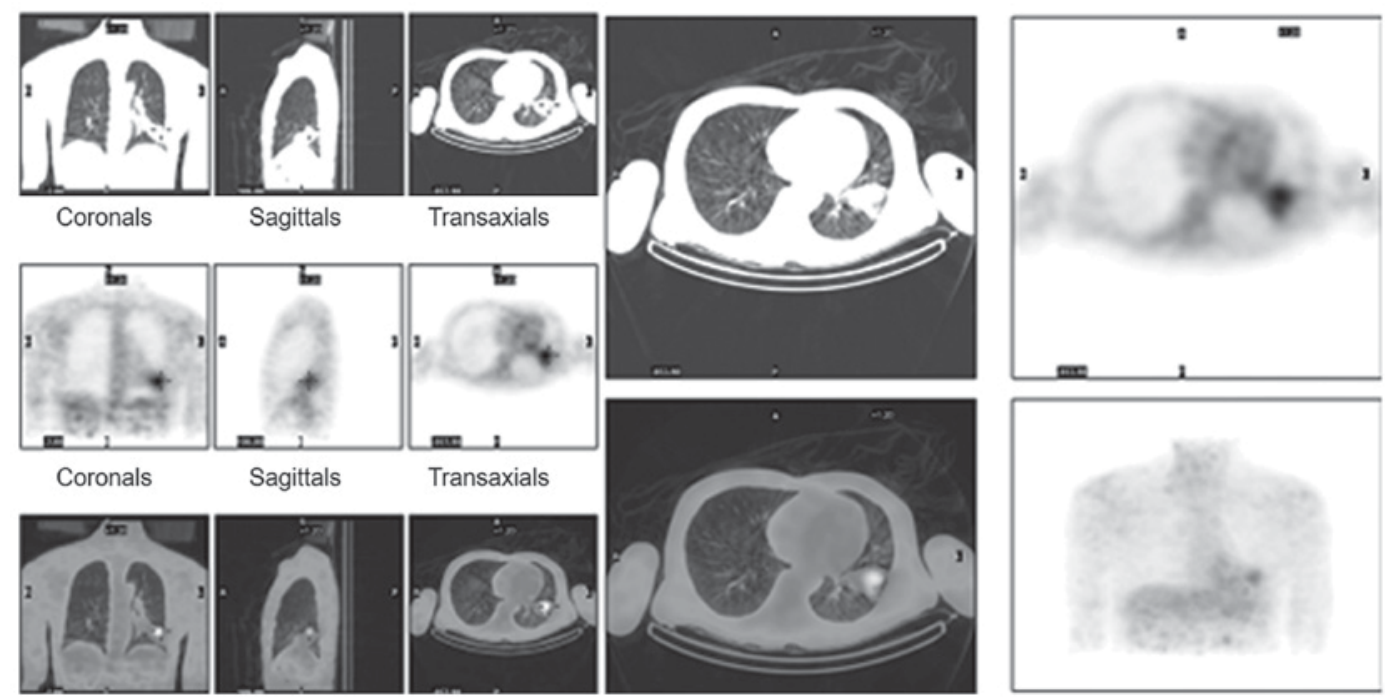

Figure 2. ${ }^{18} \mathrm{~F}$-deoxyglucose dual-head coincidence single-photon emission computerized tomography scan indicated that the maximum tumor/non-tumor was 11.63 and therefore, lung cancer should not be excluded.
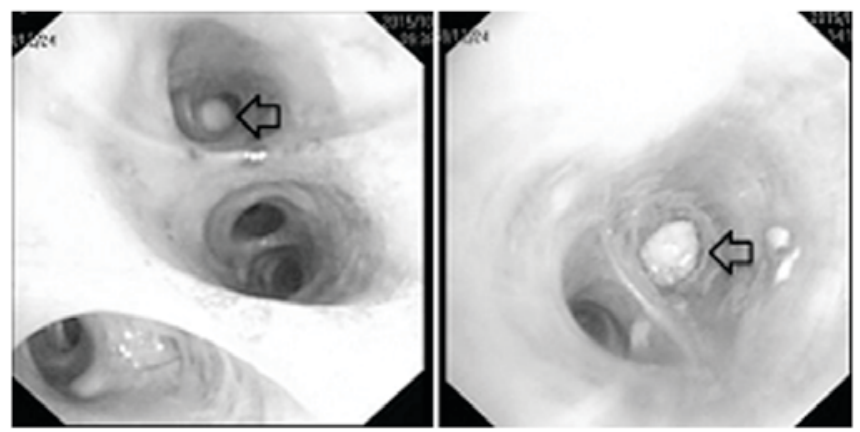

Figure 3. Fiberoptic bronchoscopy revealed a white necrotic mass in the medial anterior basal segment of left lower lobe, which completely obstructed the subsegmental bronchus (arrow).

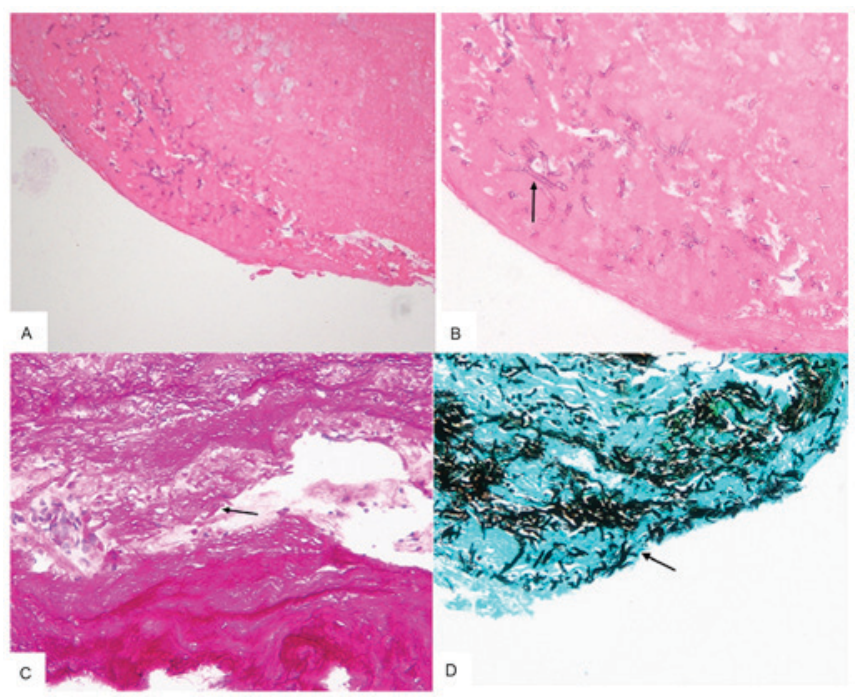

Figure 4. Histopathological examination of bronchoscopic biopsied specimen was completed using haematoxylin and eosin staining (A) at a magnification of $\mathrm{x} 100$ where hyphae like structure were observed,(B) and a magnification of x200 where sections of necrotic tissue and visible fungus hyphae with branch and sub-section were observed (black arrow). (C) Periodic acid-Schiff staining allowed the observation of red hyphae (black arrow) and (D) hexamine silver staining indicated the presence of black hyphae (black arrow).

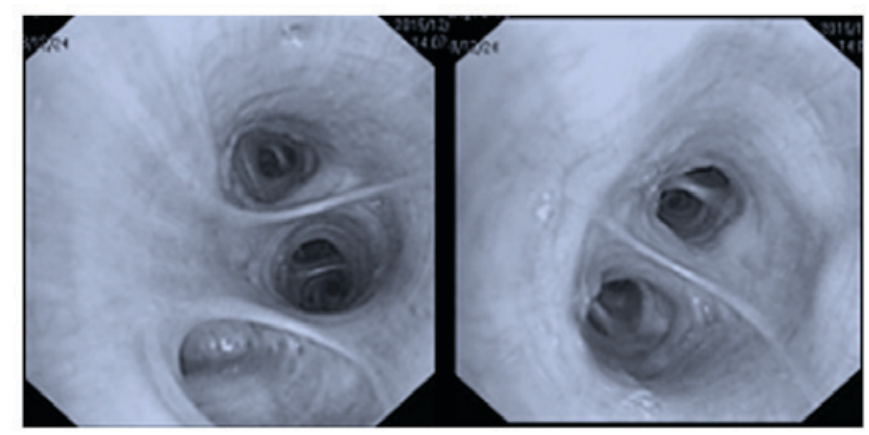

Figure 5. Following fiberoptic bronchoscopy, the patient was administered interventional voriconazole therapy for 1 month. A second bronchoscope assessment demonstrated that the lumen was completely unobstructed.

was conducted and samples were stained using silver solution for $1.5 \mathrm{~h}$ at $65^{\circ} \mathrm{C}$ first, then exposed to $0.25 \%$ chloride for $2 \mathrm{~min}, 3 \%$ sodium thiosulfate for $1 \mathrm{~min}$ and $0.1 \%$ bright green staining for $1 \mathrm{~min}$ at room temperature. Periodic acid-Schiff and hexamine silver staining results were positive (Fig. 4). The patient was then treated with oral voriconazole (Pfizer, New York, United States) $200 \mathrm{mg}$ once a day for 1 month. The lumen was reassessed with the bronchoscope and was observed to be completely unobstructed (Fig. 5). A repeat chest CT demonstrated that the left lung lesion was largely absorbed (Fig. 6). In addition, the patient's symptoms were noticeably relieved, no fever was observed, bloody sputum was no longer observed 3 days post interventional therapy. Complete blood count and routine blood chemistry markers were maintained at normal levels.

Literature comparison. By combining the present case with similar cases identified in the literature, a retrospective analysis of the clinical and radiological characteristics in addition to the bronchoscopic appearance was performed. The key word 'endobronchial aspergilloma' was searched in the PubMed NCBI database (ncbi.nlm.nih.gov/pubmed?holding=icnsibslib), by excluding cases without histopathological confirmation and 

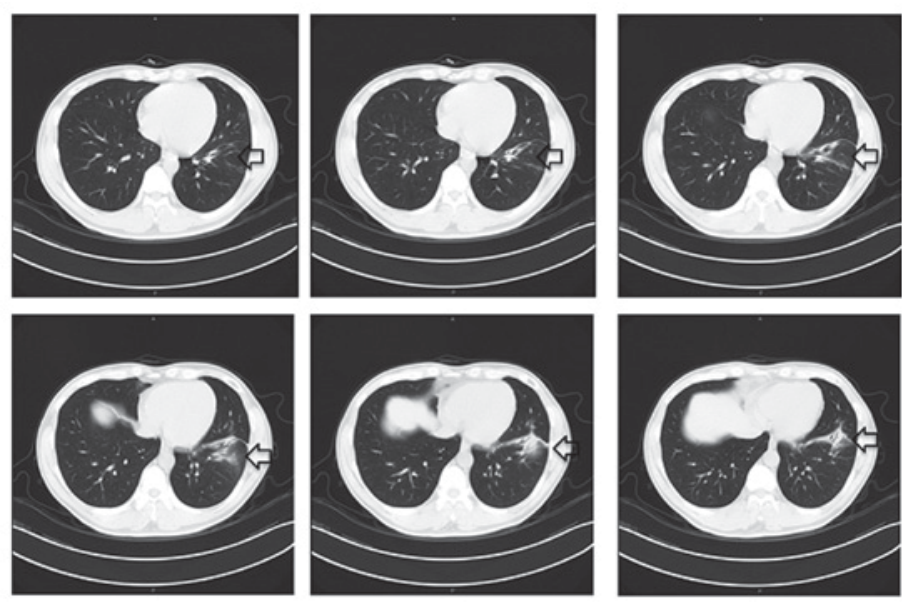

Figure 6. Following fiberoptic bronchoscopy, the patient was administered interventional voriconazole therapy for 1 month. A follow-up computed tomography scan demonstrated that the left lung lesions was absorbed.

simple Aspergilloma. In total, 17 patients meeting the EBA diagnosis criteria were included (including the case reported in the current study). The number of males $(13 / 17 ; 76.5 \%)$ was notably higher, compared with women $(4 / 17 ; 23.5 \%)$, and ages ranged from 33 to 82 years old (median age, 59). Of the 17 patients, 9 (52.9\%) were smokers. Diagnoses of previous lung diseases included pulmonary tuberculosis $(\mathrm{n}=8 ; 47.6 \%)$, lung cancer $(\mathrm{n}=4 ; 23.5 \%)$, pulmonary resection (early stage lung cancer; $\mathrm{n}=1 ; 5.9 \%)$ and foreign body of the right main bronchus $(\mathrm{n}=1 ; 5.9 \%)$. Notably, 2 patients had previously been diagnosed with endobronchial lung cancer covered by aspergillosis (patients 13 and 14). A total of 8 patients had non-pulmonary comorbidities, including dilated cardiomyopathy $(n=1)$, primary thrombocytosis $(n=1)$, intracerebral hemorrhage $(n=1)$, chronic heart failure $(n=2)$, subarachnoid hemorrhage $(n=1)$, hypertension $(n=1)$, rheumatoid arthritis $(n=1)$, hepatichemangima $(n=1)$ and diabetes $(n=4)$. None of the patients exhibited evidence of severe immune deficiency. Primary clinical symptoms were hemoptysis $(9 / 17 ; 53 \%)$, cough $(7 / 17 ; 41.2 \%)$ and dyspnea $(3 / 17 ; 17.6 \%)$. Microbiology examination was performed in the bronchial lavage fluid of 12 patients, among whom only 2 exhibited aspergilloma, and 5 patients were treated with antifungal drugs (unknown) but the treatment response was not clear, with the exception for the present patient. Clinical characteristics of the 17 patients with EBA are summarized in Table I.

X-ray examination was performed for 13 patients, and all but 1 exhibited abnormal manifestations. The abnormal region was most often in the upper lobe $(9 / 13 ; 69.2 \%)$, than in the left $(8 / 13 ; 61.5 \%)$. Primary manifestations were pulmonary fibrotic changes along with lung volume loss, similar to old pulmonary tuberculosis. Mass or nodular shadows were also detected as presentations.

CT scanning indicated that Aspergillus lesions were more likely to be identified in the left lung $(13 / 17 ; 76.5 \%)$ than in the right $(4 / 17 ; 23.5 \%)$. Lesions were most common in the left upper lobe $(6 / 17 ; 35.3 \%)$ and left lower lobe $(6 / 17$; $35.3 \%$ ) on the CT scan. The types of Aspergillus identified by chest $\mathrm{CT}$ included: Space-occupying lesions $(\mathrm{n}=10 ; 58.8 \%)$, aspergilloma $(\mathrm{n}=3 ; 17.6 \%)$, pneumonic consolidation $(\mathrm{n}=2$; $11.8 \%)$, and ground glass opacity $(\mathrm{n}=1 ; 5.9 \%)$. CT results indicated a mass with a multi-lobulated contour in 3 patients (patients 1, 10 and 15), and an endobronchial lesion, such as a foreign body or broncholithiasis, was suspected in 4 patients (patients 2, 8, 16 and 17). Multiple calcified nodules and fibrotic changes as sequelae of previous pulmonary tuberculosis were noted in 6 patients (patients 4-9).

Bronchoscopy examination identified a mass in the bronchial lumen of all 17 patients and 15 patients presented with endobronchial obstruction by necrotic material. The abnormal regions under bronchoscopy were consistent with the CT locations. Table II summarizes the radiological characteristics and the bronchoscopic appearance in 17 patients with EBA.

\section{Discussion}

Aspergilloma often occurs in lung parenchyma or the lung cavity (12). EBA is a rare space-occupying disease of the endobronchial fungi, and it may not belong to the current classification of Aspergillus spp. (IPA, CNA, ABPA and SIA). It is a substantive or cavitary lesion caused by an overgrowth of aspergilloma with a non-invasive form in endobronchial areas through inhalation and fumigation, which is always limited to the main bronchus (13). EBA may present either as the sole manifestation of pulmonary aspergillosis or with other forms of pulmonary aspergillosis. CNA and ABPA may be associated with the EBA or the visible aspergilloma inside the lung cavity, demonstrated by bronchoscopy. Among 10 cases of EBA reported by Ma et al (6), 2 patients had lung parenchymal lesions with EBA in the lung cavity.

It is not easy to directly diagnose EBA by imaging or other noninvasive tests. In the present study, all patients were diagnosed incidentally when having a bronchoscopy and this was confirmed by histological examination and culture. Only 3 patients were suspected to have Aspergillus on the basis of CT results.

Hemoptysis $(9 / 17 ; 53 \%)$ and cough $(7 / 17 ; 41.2 \%)$ were the most common symptoms of EBA, and hemoptysis specifically, may be a result of the Aspergillus colonization, which makes the diagnosis via FOB important (6). The current study suggested the detection rate of the microbiology examination using bronchial lavage fluid was low (16.7\%); therefore, 


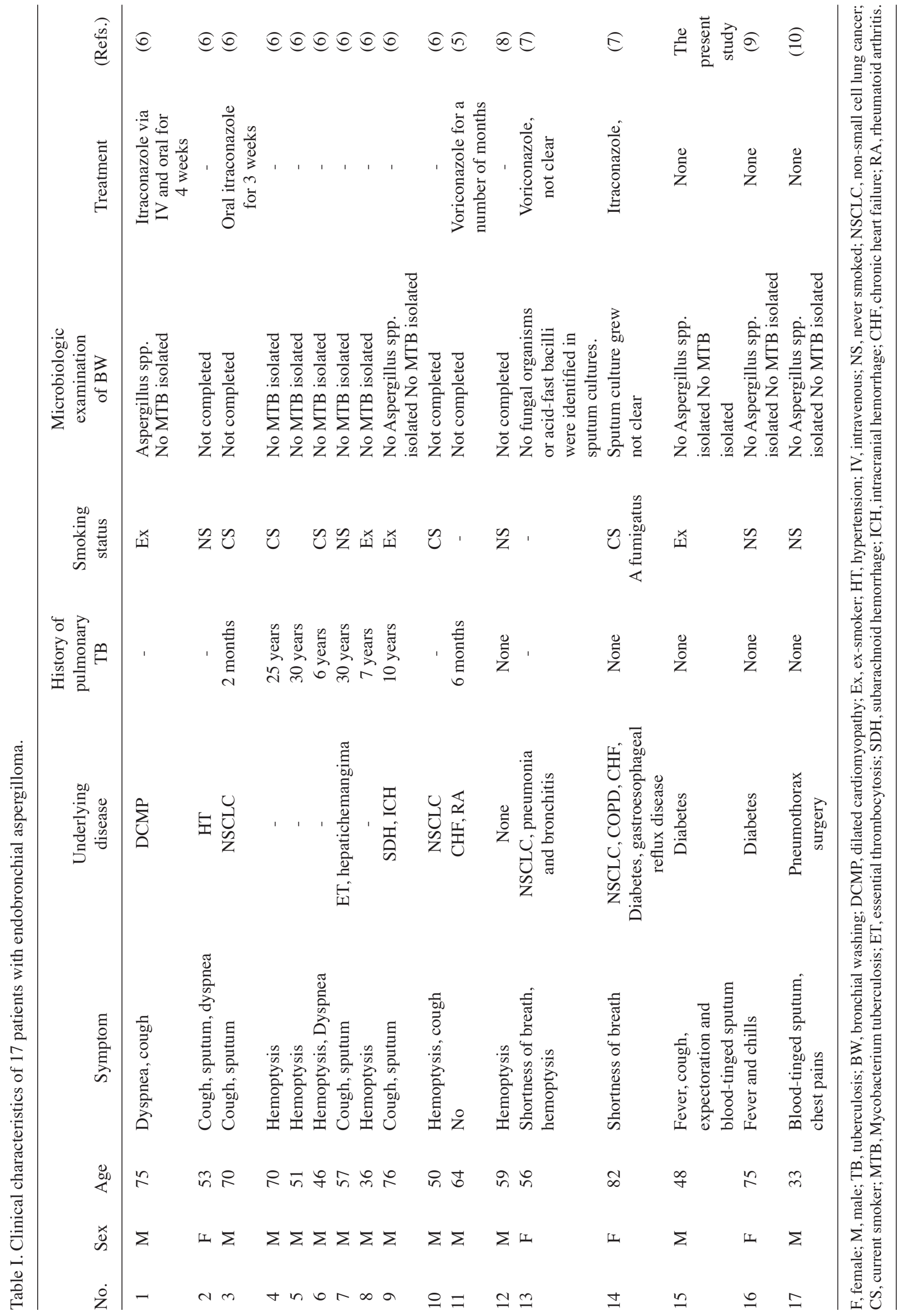




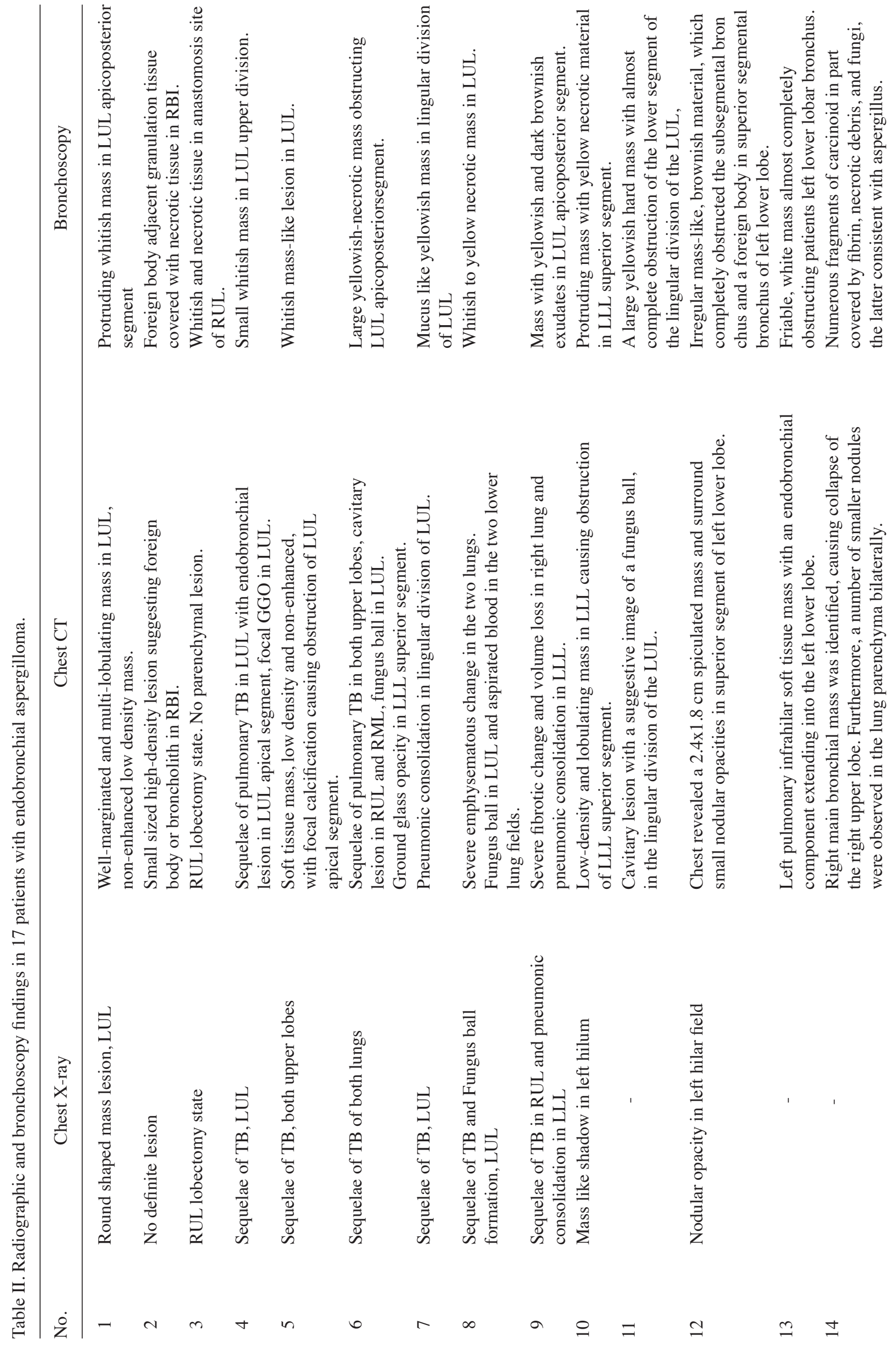


histopathological examination was required. None of the 17 cases were reported to have exhibited complications or adverse effect following biopsy.

Impaired immune function and certain comorbidities, such as active tuberculosis or diabetes, are important factors for fungal infection. Although there were no reports regarding the patients' immune status in the current study, it was revealed that of the 17 patients, 12 (70.6\%) had previously been diagnosed with lung diseases, including pulmonary tuberculosis and lung cancer, and 8 patients (47.6\%) had non-pulmonary comorbidities, such as dilated cardiomyopathy, essential thrombocytosis, cerebrovascular accident, hypertension and diabetes.

In patients with normal immune function, the formation of aspergilloma was considered to require a nest structural change that induces the airflow stasis, which helps Aspergillus in the airflow to colonize (6). In the present study, 8 patients with old pulmonary tuberculosis were included, and they exhibited destructive and fibrotic changes of the parenchyma, which potentially resulted in airway stenosis and obstruction. In addition, the majority of those who had no history of tuberculosis presented with a narrow or completely blocked lumen caused by lung cancer, lung resection, foreign bodies or granulation tissue, which was confirmed through imaging and bronchoscopy. Therefore, this change of the bronchial lumen may be considered to be an important factor to potentially induce airflow stasis and Aspergillosis colonization in bronchioles, in addition to the formation of aspergilloma. Lesions were also identified in the left lung more often than in the right, and it is suggested that this is because the left lung is anatomically closer to the heart and aorta. In addition, the left main bronchus is longer and narrower than the right side, thus it is easier to induce airflow stasis in the bronchioles of the left lung, causing EBA (13).

Notably, in the present study, 4 patients had lung cancer, of which 1 underwent surgery, 1 was affected by aspergillus (6), and the other 2 reported by Nilsson et al (7) had endobronchial tumor covered by aspergilloma. In Nilsson's report, the fungal hyphae were identified via histopathology and the patients were subsequently treated with anti-fungal drugs (unknown). However, in both the reported cases, the mass kept growing and finally the tumor was identified through further biopsy. There was no clear evidence to link Aspergillus and cancer development in the literature (7) and it is rarely reported that fungal infection may co-exist with solid types of cancer in immunocompetent patients $(14,15)$. Malignant tumor may be a factor for local fungal infection including EBA.

The optimal treatment of EBA has not yet been established. The short-term follow-up in the current study confirmed that through interventional treatment using bronchoscopy and systemic antifungal therapy, the prognosis of EBA is excellent. Nevertheless, Jung et al (8) argued that for clinically asymptomatic patients, there is no requirement for treatment, and in cases of mild hemoptysis such as in the present patient's case, medical therapy with bed rest, humidified oxygen, cough suppressants and postural drainage is sufficient (8). To the best of our knowledge, there is no consistent evidence that aspergilloma responds to systemic administration of antifungal agents (12). In certain reports, surgical treatment was a good alternative (16-18), but the pulmonary function and tolerance of patients should be considered. It is suggested that 
selecting appropriate treatments according to different conditions of patients is reasonable, and the combination of direct bronchoscopic intervention with antifungal therapy may be advantageous.

In conclusion, the dominant symptom for EBA was identified to be hemoptysis in the present analysis. Chest CT demonstrated that aspergillosis lesions were more frequently identified in the left lung compared with the right. It was also revealed that endobronchial aspergilloma often occurs in individuals with comorbidities that may potentially impair immune function and underlying lung diseases that cause lumen structural change or bronchial obstruction. Previous studies have provided sufficient evidence that endobronchial aspergilloma may be clearly diagnosed by bronchoscopy biopsy, but the potential for the co-exististing of tumors requires consideration (5-10). Finally, based on clinical experience, the combination of bronchosopic intervention and anti-fungal therapy may be an effective treatment for patients with EBA.

\section{Acknowledgements}

The abstract of this manuscript was presented at the ERS International Congress 3rd-7th September 2016 in London, UK and published as abstract no. PA3715 in the European Respiratory Journal 48 (suppl 60): 2016.

\section{References}

1. Silva EF, Barbosa Mde P, Oliveira MA, Martins RR and Fontinele e Silva J: Chronic necrotizing pulmonary aspergillosis. J Bras Pneumol 35: 95-98, 2009 (In English, Portuguese).

2. Hospenthal DR, Kwon-Chung KJ and Bennett JE: Concentrations of airborne aspergillus compared to the incidence of invasive aspergillosis: Lack of correlation. Med Mycol 36: 165-168, 1998

3. Zmeili OS and Soubani AO: Pulmonary aspergillosis: A clinical update. QJM 100: 317-334, 2007.

4. Chabi ML, Goracci A, Roche N, Paugam A, Lupo A and Revel MP: Pulmonary aspergillosis. Diagn Interv Imaging 96: 435-442, 2015.
5. Araújo D, Figueiredo M and Monteiro P: Endobronchial aspergilloma: An unusual presentation of pulmonary aspergillosis. Rev Port Pneumol (2006) 22: 61-62, 2016.

6. Ma JE, Yun EY, Kim YE, Lee GD, Cho YJ, Jeong YY, Jeon KN, Jang IS, Kim HC, Lee JD and Hwang YS: Endobronchial aspergilloma: Report of 10 cases and literature review. Yonsei Med J 52: 787-792, 2011.

7. Nilsson JR, Restrepo CS and Jagirdar J: Two cases of endobronchial carcinoid masked by superimposed aspergillosis: A review of the literature of primary lung cancers associated with aspergillus. Ann Diagn Pathol 17: 131-136, 2013.

8. Jung SW, Kim MW, Cho SK, Kim HU, Lee DC, Yoon BK, Jeong JP and Ko YC: A case of Endobronchial aspergilloma associated with foreign body in immunocompetent patient without underlying lung disease. Tuberc Respir Dis (Seoul) 74: 231-234, 2013.

9. Yeo CD, Baeg MK and Kim JW: A case of endobronchial aspergilloma presenting as a broncholith. Am J Med Sci 343: 501-503, 2012.

10. Kim JS, Rhee Y, Kang SM, Ko WK, Kim YS, Lee JG, Park JM, Kim SK, Kim SK, Lee WY and Chang J: A case of endobronchial aspergilloma. Yonsei Med J 41: 422-425, 2000.

11. British Thoracic Society Bronchoscopy Guidelines Committee, a Subcommittee of the Standards of Crae Committee of the British Thoracic Society: British thoracic society guidelines on diagnostic flexible bronchoscopy. Thorax 56 (Suppl I): i1-i21, 2001.

12. Patterson KC and Strek ME: Diagnosis and treatment of pulmonary aspergillosis syndromes. Chest 146: 1358-1368, 2014.

13. Soubani AO and Chandrasekar PH: The clinical spectrum of pulmonary aspergillosis. Chest 121: 1988-1999, 2002.

14. Tashiro T, Izumikawa K, Tashiro M, Takazono T, Morinaga Y, Yamamoto K, Imamura Y, Miyazaki T, Seki M, Kakeya H, et al: Diagnostic significance of aspergillus species isolated from respiratory samples in an adult pneumology ward. Med Mycol 49: 581-587, 2011.

15. Ohmagari N, Raad II, Hachem R and Kontoyiannis DP: Invasive aspergillosis in patients with solid tumors. Cancer 101: 2300-2302, 2004.

16. Stevens DA, Kan VL, Judson MA, Morrison VA, Dummer S, Denning DW, Bennett JE, Walsh TJ, Patterson TF and Pankey GA: Practice guidelines for diseases caused by Aspergillus. Infectious diseases society of america. Clin Infect Dis 30: 696-709, 2000

17. Sagan D and Goździuk K: Surgery for pulmonary aspergilloma in immunocompetent patients: No benefit from adjuvant antifungal pharmacotherapy. Ann Thorac Surg 89: 1603-1610, 2010.

18. Lee JG, Lee CY, Park IK, Kim DJ, Chang J, Kim SK and Chung KY: Pulmonary aspergilloma: Analysis of prognosis in relation to symptoms and treatment. J Thorac Cardiovase Surg 138: 820-825, 2009. 